\title{
À la recherche d'un statut professionnel dans le champ paramédical. Le cas de la diététique, de la physiothérapie et de la technologie médicale (1940-1973)
}

\section{Lucie Piché et Nadia Fahmy-Eid}

Volume 45, numéro 3, hiver 1992

URI : https://id.erudit.org/iderudit/304991ar

DOI : https://doi.org/10.7202/304991ar

Aller au sommaire du numéro

Éditeur(s)

Institut d'histoire de l'Amérique française

ISSN

0035-2357 (imprimé)

1492-1383 (numérique)

Découvrir la revue

Citer cet article

Piché, L. \& Fahmy-Eid, N. (1992). À la recherche d'un statut professionnel dans le champ paramédical. Le cas de la diététique, de la physiothérapie et de la technologie médicale (1940-1973). Revue d'histoire de l'Amérique française, 45(3), 375-401. https://doi.org/10.7202/304991ar
Résumé de l'article

Cet article examine les luttes menées par les associations regroupant les diététistes, les physiothérapeutes et les technologistes médicales pour faire reconnaître le statut professionnel de leurs membres. Après avoir examiné, lors d'étapes antérieures de notre recherche, divers aspects relatifs à la formation dans les trois disciplines concernées, nous analysons dans cet article les stratégies développées par les diverses associations face à l'État, aux administrations hospitalières et au corps médical afin d'obtenir ce statut professionnel. Ce faisant, nous visons à souligner la complexité, mais aussi la dimension politique que présente la professionnalisation dans le champ de la santé. Plus particulièrement, l'intervention de l'État dans ce champ dès le début des années 1960 constitue, selon nous, un révélateur important des rapports de pouvoir qui y prévalent. 


\title{
À LA RECHERCHE D'UN STATUT PROFESSIONNEL DANS LE CHAMP PARAMÉDICAL LE CAS DE LA DIÉTÉTIQUE, DE LA PHYSIOTHÉRAPIE ET DE LA TECHNOLOGIE MÉDICALE (1940-1973)
}

\author{
LUCIE PICHÉ \\ NADIA FAHMY-EID \\ Département d' histoire \\ Université du Québec à Montréal
}

\section{RÉSUMÉ}

Cet article examine les luttes menées par les associations regroupant les diététistes, les physiothérapeutes et les technologistes médicales pour faire reconnaître le statut professionnel de leurs membres. Après avoir examiné, lors d'étapes antérieures de notre recherche, divers aspects relatifs à la formation dans les trois disciplines concernées, nous analysons dans cet article les stratégies développées par les diverses associations face à l'État, aux administrations hospitalières et au corps médical afin d'obtenir ce statut professionnel. Ce faisant, nous visons à souligner la complexité, mais aussi la dimension politique que présente la professionnalisation dans le champ de la santé. Plus particulièrement, l'intervention de l'État dans ce champ dès le début des années 1960 constitue, selon nous, un révélateur important des rapports de pouvoir qui y prévalent.

\begin{abstract}
This paper examines the struggles waged by organizations of dietitians, physiotherapists and laboratory technologists for recognition of their members' professional status. Following earlier research on various aspects of academic training in these three disciplines, this study examines the strategies which these professional associations pursued in relation to the State, the medical profession and hospital administrations in order to achieve their goal. Our purpose is to emphasize both the complexity and the political dimension of the professionalization process in the field of health care. More specifically, we believe that State intervention from the early 1960's sheds significant light on the balance of power prevailing in this field.
\end{abstract}

Tout au long du $\mathrm{XX}^{\mathrm{e}}$ siècle, le champ socio-sanitaire québécois connaît de multiples transformations qui modifient en profondeur les processus de soin et de guérison et entraînent, notamment, une spécialisation croissante. Ainsi, autour du médecin et à côté de l'infirmière se greffe progressivement un personnel de plus en plus diversifié dont les tâches ne cesseront de se complexifier. L'existence 
de ce processus est d'ailleurs consacrée, au mitan du $\mathrm{XX}^{\mathrm{e}}$ siècle, $\mathrm{par}$ l'apparition d'un nouveau vocable, soit celui de personnel paramédical. Les membres de ces nouvelles catégories d'emploi chercheront rapidement à s'organiser sur une base professionnelle afin de faire reconnaître leurs compétences et de délimiter leur champ d'exercice respectif. Notre étude vise à retracer l'évolution de ce processus de professionnalisation à travers l'analyse des luttes menées à cet égard, entre 1940 et 1973, par les associations de trois professions témoins: celles de diététique, de physiothérapie et de technologie médicale ${ }^{1}$.

Ces professions présentent plusieurs traits communs. Elles émergent et se développent dans l'entre-deux-guerres et reposent, dès le milieu des années 1940, sur une formation universitaire. Elles recrutent, de plus, une main-d'œuvre majoritairement, si ce n'est exclusivement, féminine puisqu'elles figurent parmi les principales orientations suggérées aux filles qui désiraient poursuivre des études supérieures dans le champ de la sante. Cette formation universitaire ne permet cependant pas d'accéder, de facto, à l'univers professionnel auquel prédispose, en principe, un tel apprentissage. Les nouvelles catégories d'emploi devront d'abord faire reconnaître le caractère professionnel des services de leurs membres, tant au niveau de la nature des actes posés que de la rétribution qui y correspond; une démarche qui devra être effectuée non seulement auprès des institutions hospitalières et des autorités gouvernementales, mais aussi auprès des médecins puisque ces derniers occupent, depuis la fin du $\mathrm{XIX}^{\mathrm{e}}$ siècle, le sommet de la pyramide du savoir et du pouvoir dans le champ socio-sanitaire. Notre propos consistera ici à identifier les outils dont disposent ces praticiennes de la santé pour atteindre leurs objectifs, les stratégies qu'elles mettent en œuvre, ainsi que le type d'obstacles auxquels elles font face. Une telle analyse permettra de mieux circonscrire toute la complexité du processus de professionnalisation du personnel paramédical et de mettre en relief les principaux enjeux qui y sont liés.

L'évolution des professions paramédicales et l'analyse des dynamiques qui modulent leur insertion dans le champ socio-sanitaire n'ont pas fait l'objet de recherches historiques nombreuses ${ }^{2}$. À partir

1 Ce projet est subventionné par le Conseil de recherches en sciences humaines du Canada dans le cadre des subventions stratégiques sur le sujet «Femmes et travail».

2 La profession d'infirmière fait toutefois figure d'exception. Pour les États-Unis, on pourra consulter notamment B. Melosh, The Physician's Hand. Work Culture and Conflict in American Nursing (Philadelphia, Temple University Press, 1982); C. Davies (ed.), Rewriting Nursing History (Totowa, New Jersey, Barnes and Noble Books, 1980); S. M. Reverby, Ordered to Care. The Dilemma of American Nursing, 1850-1945 (Cambridge, University Press of Cambridge, 1988). Pour le Canada et le Québec, voir notamment M. Q. Innis (ed.), Nursing 
des années 1940, ces professions occupent pourtant une place de plus en plus importante dans l'univers hospitalier. L'éclatement des connaissances bio-médicales et l'usage d'appareils de diagnostic et de traitement toujours plus perfectionnés a entraîné, en effet, une spécialisation croissante de la main-d'œuvre soignante et l'émergence de nouvelles pratiques dans le domaine de la santé. L'augmentation du volume des soins, au lendemain de la Seconde Guerre mondiale, accentuera par ailleurs ce phénomène: si l'ensemble du personnel hospitalier quadruple entre 1953 et 1967, les effectifs infirmiers sont, quant à eux, multipliés par six, pendant que ceux du personnel professionnel et technique augmentent de $700 \%^{3}$. En 1952, 77 diététistes, 38 physiothérapeutes et 353 technologistes de laboratoire travaillent ainsi dans les institutions hospitalières. En 1966, on y recense 264 diététistes, 361 physiothérapeutes et 1583 technologistes $^{4}$. Le travail du personnel affecté à ces tâches sera fortement encadré par les médecins qui s'assureront ainsi du contrôle du processus thérapeutique. Afin de mieux comprendre les fondements d'une telle division du travail, de même que son impact sur l'obtention d'un statut professionnel, il peut s'avérer utile de se référer ici à un modèle d'interprétation développé par la sociologie des professions.

Selon les normes traditionnelles établies par les professions libérales, est professionnelle la personne qui a acquis un bagage de connaissances théoriques spécialisées, qui jouit d'une autonomie dans sa pratique, dont la rémunération est établie de gré à gré et dont la profession comporte une éthique de service. Ces normes, qui résultent d'un construit socio-historique, s'imposent au $\mathrm{XIX}^{\mathrm{e}}$ siècle dans bon nombre de sociétés occidentales ${ }^{5}$. Elles constituent, affirme $M$. Sarfatti-Larson, une forme de clôture sociale («social closure») qui confère aux groupes auxquels elle s'applique un prestige et des

Education in a Changing Society (Toronto, University of Toronto Press, 1970); Y. Cohen et M. Dagenais, «Le métier d'infirmière: savoirs féminins et reconnaissance professionnelle», Revue d'histoire de l'Amérique française, 41,2 (automne 1987): 155-177; J. Daigle, Devenir infirmière: le système d'apprentissage et la formation professionnelle à l'Hôtel-Dieu de Montréal - 1920 à 1970, thèse de Ph.D. (histoire), Université du Québec à Montréal, 1990; André Petitat, Les infirmières, de la vocation à la profession (Montréal, Boréal Express, 1989).

$3 \mathrm{R}$. Béland et T. Boudreau, La prévision de la main-d'auvre dans le secteur hospitalier, Annexe 8 de la Commission d'enquête sur la santé et le bien être-social, Québec, 1970, 90-91.

$4 \quad$ Ibid., 159 et 165.

5 Il faut préciser ici que c'est dans les sociétés nord-américaines que ce modèle s'est davantage développé. Voir notamment à ce sujet A. Abbot, The System of Professions (Chicago, The University of Chicago Press, 1988). E. Friedson, The Profession of Medecine. A Study of the Sociology of Applied Knowledge (New York, Dodd, Mead \& Co., 1972). M. Sarfatti-Larson, The Rise of Professionnalism. A Sociological Analysis (Berkeley, University of California Press, 1977). 
privilèges certains, d'où la tendance de ces groupes à perpétuer de telles normes ${ }^{6}$. Ils le feront surtout en obtenant de l'État un statut de corporation fermée. Ce statut confère en effet au groupe qui l'acquiert le monopole de son titre, de même que le droit exclusif de poser un certain nombre d'actes professionnels ${ }^{7}$.

Le personnel affecté aux nouveaux emplois qui émergent au $\mathrm{XX}^{\mathrm{e}}$ siècle dans l'univers sanitaire va rapidement chercher à s'organiser en fonction de ces paramètres, mais plusieurs facteurs contrecarreront leurs aspirations. Ces emplois sont, en premier lieu, issus d'une division du travail sanitaire ou d'une délégation d'actes professionnels qui les confinent dans des univers de savoirs et de pratiques bien délimités, ce qui réduit considérablement l'étendue de leurs interventions et limitent d'autant leur autonomie. De plus, leur formation, de plus courte durée que celle des médecins, repose certes sur des connaissances théoriques, mais elle comporte une part importante de savoirs pratiques, ce qui s'avère moins rentable dans la hiérarchie des savoirs et des pouvoirs ${ }^{8}$. Le corps médical bénéficie pour sa part d'un pouvoir depuis longtemps bien établi et d'un statut social prestigieux que les nouvelles professions paramédicales peuvent difficilement concurrencer'. À ces obstacles s'ajoute le fait que la majorité du personnel paramédical est salariée et travaille, dans l'univers hospitalier, sous la gouverne d'un employeur qui intervient dans la définition des tâches et le régime de travail auquel est soumis ce personnel. Ces divers éléments apparaissent, à plusieurs égards, difficilement compatibles avec le modèle des professions libérales ${ }^{10}$.

On peut se demander, enfin, si les clivages sexuels qui caractérisent la division du travail en milieu hospitalier tout au long du $\mathrm{XX}^{\mathrm{e}}$

6 M. Sarfatti-Larson, «À propos des professionnels et des experts - ou comme il est peu utile d'essayer de tout dire», Sociologie et sociétés, 20,2 (octobre 1988): 23-40.

7 Lui est également reconnu le droit de contrôler la formation et l'inscription de ses membres ainsi que le droit de juger de la qualité de leurs actes.

8 L'analyse des programmes de formation de diététique, de physiothérapie et de technologie médicale à l'Université de Montréal et à l'Université McGill a révélé l'existence d'un contrôle constant de la part du corps médical sur les cursus de ces trois disciplines. Voir à ce sujet Nadia Fahmy-Eid et Aline Charles, "Savoir contrôlé ou pouvoir confisqué? La formation des filles en technologie médicale, réhabilitation et diététique (1940-1970)", Recherches féministes, 1,1 (1988): 5-30; Nadia Fahmy-Eid et Johanne Collin, «Savoir et pouvoir dans l'univers des disciplines paramédicales: la formation en physiothérapie et en diététique à l'Université McGill, 1940-1970», Histoire sociale - Social History, 22,43 (mai 1989): 35-63.

9 Voir notamment à ce sujet G. Dussault, «La régulation des professions sanitaires», dans le numéro déjà cité de Sociologie et sociétés.

10 On pourra notamment consulter à ce sujet G. Dussault, «Professionnalisation et déprofessionnalisation», J. Dufresne et al., Traité d'anthropologie médicale (Québec, Presses de l'Université du Québec, 1985), 605-616; A. Etzioni, The Semi-Professions and their Organizations. Teachers, Nurses, Social Workers (États-Unis, Free Press, 1969); T. Hallyday, «Professions, Class, Capitalism», Archives Européennes de sociologie, 24 (1983): 321-346. 
siècle ne doivent pas s'ajouter aux obstacles auxquels fait face le personnel paramédical ${ }^{11}$. Alors que des professions prestigieuses telles que la médecine, la pharmacie ou la dentisterie sont restées longtemps masculines, celles qui sont nées dans le sillage de la division du travail sanitaire ont été, bien souvent au départ, des emplois féminins. Il s'avère donc important de s'interroger sur l'incidence des rapports sociaux de sexe sur le processus de professionnalisation du personnel paramédical.

L'analyse des politiques qu'ont élaborées les associations de diététique, de physiothérapie et de technologie médicale, entre 1940 et 1973, pour faire reconnaître le caractère professionnel des services dispensés par leurs membres nous permettra de mieux circonscrire toute la complexité du processus de professionnalisation du personnel paramédical et la nature des enjeux qui y sont liés. Nous privilégierons ici deux angles d'approche. Dans un premier temps, nous examinerons la nature des démarches entreprises par ces associations auprès des administrations hospitalières et de l'État afin d'améliorer les conditions de travail de leurs membres. Quels types de stratégies mettentelles en œuvre pour atteindre leurs objectifs et est-ce que l'intervention de l'État dans la gestion et le financement des hôpitaux a une incidence particulière sur ces stratégies? Dans quelle mesure, par exemple, la syndicalisation massive du personnel hospitalier influence-t-elle la ligne de conduite des associations concernées? Comment s'adaptent-elles à un nouveau contexte où l'employeur ultime qu'est devenu l'État est également l'arbitre suprême de la négociation?

Dans un deuxième temps, nous examinerons les politiques élaborées pour obtenir de l'État une reconnaissance professionnelle légale et s'assurer, en particulier, de l'octroi d'un statut de corporation fermée. Cette reconnaissance suppose cependant que soient définis le champ d'exercice, la nature des actes posés, de même que le degré d'autonomie dont disposent les travailleuses dans leur pratique. En raison de sa position dans la hiérarchie socio-sanitaire, le corps médical constitue à cet égard un interlocuteur supplémentaire. Il s'agira d'examiner si les revendications de ces associations sont com-

11 La corporation des diététistes du Québec ne compte que trois hommes pour 514 femmes en 1970. En 1969, l'association québécoise regroupant les physiothérapeutes ne comptent que 15 hommes sur un total de 312 membres (soit moins de 5\%). La technologie médicale offre un taux de masculinité plus élevé: en 1971, le rapport est de 245 hommes pour 1264 femmes. Ces données ont été compilées à partir des registres de membres des corporations québécoises. Voir également R. Boulard, Recueil de données statistiques sur la main-d'auvre de niveau syndicable du réseau des Affaires sociales en 1977-1978 (Québec, Ministère des Affaires sociales, 1981). 
patibles avec l'organisation du travail dans l'univers hospitalier et dans quelle mesure l'État et les médecins ont intérêt à modifier, et surtout à élargir, les frontières du modèle professionnel traditionnel hérité des professions libérales pour y inclure les nouvelles catégories d'emploi occupées par les travailleuses du paramédical. L'examen des archives des associations canadiennes et québécoises de diététique, de physiothérapie et de technologie médicale nous permettra de répondre à ces questions ${ }^{12}$.

\section{I - DE LA POLITIQUE ASSOCIATIVE À L'OPTION SYNDICALE}

La création d'associations pancanadiennes constitue le premier instrument dont se dotent les nouvelles praticiennes de la santé pour promouvoir le développement de leurs professions. L'Association canadienne de diététique (ACD) est ainsi mise sur pied en 1935, ainsi que l'Association canadienne de physiothérapie (ACP). L'Association canadienne des technologistes de laboratoire (ACTL) verra le jour deux ans plus tard, soit en $1937^{13}$. Des associations provinciales seront par la suite créées. La Corporation des diététistes du Québec (CDQ) naît en 1956, le regroupement des Physiothérapeutes de la Province de Québec Inc. (PPQ) en 1961 et, trois ans plus tard, l'Association des technologistes médicaux du Québec (ATMQ) ${ }^{14}$. La question des conditions de travail auxquelles sont soumises ces praticiennes de la santé au sein de l'univers hospitalier mobilise une part importante de l'énergie de leurs associations ${ }^{15}$. L'analyse de leurs revendications révèle l'existence d'objectifs similaires qui visent notamment à obte-

12 Ce corpus se compose principalement des journaux et des bulletins de ces regroupements et, dans le cas des associations québécoises, de documents tels les procès-verbaux, les registres des membres, des résultats d'enquêtes internes ou encore des mémoires soumis aux autorités gouvernementales.

13 Ces associations seront d'abord connues sous leur patronyme anglais, soit la Canadian Dietetic Association, la Canadian Physiotherapy Association et la Canadian Association of Laboratory Technologists. En 1944, l'ACD compte déjà 628 membres, l'ACP, 296 et l'ACTL, 506. Ces informations sont tirées des journaux de ces trois associations.

14 Les associations québécoises de physiothérapie et de technologie médicale sont incorporées en vertu de la $3 \mathrm{e}$ partie de la Loi des compagnies alors qu'en diététique, c'est la loi régissant les corporations professionnelles qui s'applique; les diététistes obtiennent en effet un statut de corporation dès 1956. Précisons par ailleurs que la section montréalaise de l'ACTL existait depuis 1958 et celle de l'ACP, depuis 1940. Les diététistes étaient quant à elles regroupées dans une section locale de l'ACD depuis 1924, sous le nom de «Quebec Dietetic Association». Elles conserveront d'ailleurs cette appellation jusqu'en 1964. Rappelons ici que la CDQ compte 483 membres en 1966 et 598 en 1970 . La PPQ regroupe pour sa part 283 membres en 1966 et 418 en 1970. L'ATMQ compte quant à elle 593 membres en 1966 et 1509 en 1971 (Registres des membres).

15 Il faut souligner ici que l'organisation et l'amélioration des programmes d'enseignement destinés aux futures diplômées mobilisera également les énergies de ces associations. 
nir une certaine adéquation entre leurs conditions de travail et le caractère professionnel des services qu'elles dispensent. L'intervention de l'État dans le champ de la santé les obligera cependant à modifier leurs stratégies et à opter pour la mise sur pied d'associations syndicales.

\section{LES DIÉTÉTISTES}

L'Association canadienne de diététique (ACD) se préoccupe du régime de travail de ses membres dès le début des années 1940 lorsqu'elle constate que plusieurs étudiantes, attirées par des salaires plus élevés, optent finalement pour des emplois qui ne sont pas directement liés à leur formation première ${ }^{16}$. Ce n'est cependant qu'au cours de la décennie suivante que l'association va s'attarder plus longuement sur cette question. Une profonde insatisfaction règne en effet parmi les diététistes œuvrant en milieu hospitalier ${ }^{17}$. Une enquête effectuée en 1956 par l'ACD révèle d'ailleurs que le salaire médian est de $3250 \$$ dans les petits hôpitaux, de 3350 dans les hôpitaux de grande taille, alors qu'il s'élève à $3500 \$$ dans les agences sociales et à $4000 \$$ dans les grandes cuisines commerciales ${ }^{18}$. Les années d'expérience et les responsabilités ne sont pas reconnues en milieu hospitalier et les salaires plafonnent rapidement. En 1965, une nouvelle enquête nationale indique que $30 \%$ des diététistes œuvrant dans les hôpitaux gagnent moins de $5000 \$$ par année alors que cette proportion chute à $9 \%$ lorsqu'on tient compte seulement de la rémunération de celles qui travaillent en milieu non hospitalier ${ }^{19}$. Une telle distorsion résulte, soulignent les auteures de l'enquête du fait que: «[The]

Cette priorité éducative dénote, comme nous l'avons démontré ailleurs, une volonté explicite et soutenue de faire reposer le professionnalisme des services offerts par les membres de chacune de ces professions, sur une formation universitaire de haut calibre. Voir à ce sujet Nadia FahmyEid et Lucie Piché, «Le savoir négocié. Les stratégies des associations de technologie médicale, de physiothérapie et de diététique pour l'accès à une meilleure formation professionnelle (19301970)», Revue d' histoire de l'Amérique française, 43,4 (printemps 1990): 509-534.

16 Ces autres voies ne sont cependant pas précisées. «Annual Reports and Proceedings - Professionnal Education», Journal of Canadian Dietetic Association (JCDA), 6,3 (décembre 1944): 93.

17 Voir à ce sujet I. MacArthur, «My Questionnaire - Your Answers - The Results», JCDA, 14,2 (septembre 1952): 29. Voir aussi «The Job Evaluation Committee Present», 18,1 (janvier 1956): 9. La diététique est, des trois professions retenues, la seule dont les membres ne travaillent pas massivement dans les hôpitaux. Elles seront engagées, dès le début du $\mathrm{XX}^{\mathrm{e}}$ siècle, dans les cuisines des grands restaurants et des magasins de grande surface et dans les cafétérias des institutions bancaires ou universitaires.

18 «A Salary Survey - The Means to an End», JCDA, 19,1 (janvier 1957): 24. Une partie des résultats de cette enquête a également été publiée dans le 18,4 (juillet 1956): 14-20.

19 E. Upton et M. Clark, «Status of Members of the Canadian Dietetic Association as Determined by Salary Surveys in 1956 and 1965», JCDA, 27,1 (novembre 1966): 26. 
salaries for dietitians in industry are often regulated by management policy and depend upon the responsabilities involved and the number of personnel in the department. No such policy appears to exist in hospitals where the salary is a matter for discussion between the administrator and the dietitian. $»^{20}$

Les enquêtes effectuées au début des années 1960 par la Corporation des diététistes québécoises (CDQ) révèlent que le Québec ne fait pas exception à la règle ${ }^{21}$. Afin de faire reconnaître la valeur du travail de ses membres, cet organisme table notamment sur le niveau de formation des diététistes. Il demande, dès 1963, la parité salariale avec les infirmières qui détiennent un baccalauréat en nursing 22 . Devant le Comité des relations de travail du ministère de la Santé la corporation exigera, cinq ans plus tard, qu'une scolarité égale débouche sur des salaires comparables. Les biochimistes et le personnel spécialisé en éducation physique ont en effet obtenu, lors de la signature de leur dernière convention collective, un salaire plus élevé que les diététistes (5 800\$ contre 5500 ) alors que la scolarité de ces dernières compte une année de plus (16 ans). Les infirmières, qui possèdent quinze années de scolarité, reçoivent pour leur part un salaire initial de $6500 \$$. Les pharmaciens gagnent, quant à eux, $6400 \$$ mais possèdent, comme les diététistes, seize années de scolarité ${ }^{23}$.

Se référant à ces ententes salariales, la corporation recommande que le salaire initial d'une diététiste professionnelle travaillant dans un établissement hospitalier soit de $6730 \$$, que la progression salariale s'échelonne sur une période de dix ans et qu'un supplément salarial soit alloué à celles qui assument des responsabilités au sein de leur

20 Ibid., 29. L'enquête de 1965 révèle par ailleurs que les salaires payés aux diététistes demeurent inférieurs à ceux que reçoivent d'autres professions reposant sur une formation similaire et exigeant le même type de responsabilités. Le rapport ne précise pas ces professions mais affirme tirer ses données du National Employment Service. Anticipated Requirements and Rates Pay for 1956 University Graduates, Part 2, mars 1956.

21 Voir à ce sujet «Report of Salary Survey Committee», Bulletin de la $C D Q, 9$ (mai 1962): 11 .

22 Michel Lapalme, "Les diététistes disent avoir droit à un salaire supérieur aux infirmières», La Presse, 12 novembre 1963, n.p. La corporation ne réussit cependant pas à convaincre l'Association des hôpitaux du Québec de la pertinence de ses demandes. Au lendemain de ces négociations infructueuses, la corporation déclare que «L'âge de notre profession et le nombre de membres qu'elle comporte comparé à la profession de nursing ne justifient peut-être pas une hausse trop considérable du salaire initial». «Rapport du Comité de l'étude des salaires», Rapport annuel - Annual Report, 1962-1963, n.p.

23 «Tableau comparatif des salaires et (de la) scolarité requise pour diverses professions paramédicales» et «Description des tâches des diététistes dans les hôpitaux et échelle des salaires minima pour 1968» (Appendice II), Bulletin de la $C D Q, 8,5$ (juin 1968). Il y a tout lieu de croire que les infirmières bénéficient des retombées des luttes syndicales qu'elles ont menées, notamment depuis le début des années 1960. 
département (700\$), de même qu'à celles qui détiennent une maîtrise (1 000\$). En 1970, l'Assurance hospitalisation impose de nouvelles échelles salariales qui, soutient la CDQ, s'avèrent toujours «nettement insuffisant(es) considérant (les) années de scolarité et (les) responsabilités» des diététistes ${ }^{24}$.

La CDQ ne semble donc pas disposer d'un pouvoir de négociation adéquat pour faire valoir ses revendications. La centralisation de la gestion de la main-d'œuvre hospitalière aux mains d'un État devenu employeur incite bientôt les diététistes à opter pour la voie syndicale, à l'instar de la majorité du personnel hospitalier. Une telle option aurait été impensable quelques années plus tôt, la syndicalisation allant à l'encontre des aspirations corporatistes des diététistes. Dès 1948, l'ACD avait fait pression auprès du ministère fédéral du Travail afin d'exclure les diététistes du personnel syndicable ${ }^{25}$. En 1960, la corporation québécoise réitère cette demande auprès des autorités provinciales $^{26}$. L'adoption du Code du travail québécois, en 1964, l'oblige cependant à revoir ses positions puisque la main-d'œuvre hospitalière dispose maintenant du droit de grève. La première ronde de négociations du secteur public et parapublic de 1964 permet au personnel des hôpitaux d'obtenir des gains appréciables et indique qu'une ère nouvelle se dessine dans les relations de travail. La CDQ semble cependant hésiter à joindre les rangs des syndicats existants. Elle soumet ainsi au ministère du Travail un amendement au Code du travail qui «permettrait aux diététistes d'être parmi les professions dont les membres forment obligatoirement un groupe distinct pour fin d'accréditation comme le sont les médecins, les avocats, les architectes, les infirmières» ${ }^{27}$. De plus, lorsqu'un comité est chargé, en 1966, d'étudier les modalités d'une éventuelle syndicalisation, la présidente de la corporation prend soin de préciser que cette initiative ne signifie pas «qu'à l'heure actuelle, la Corporation approuve nécessairement la participation à des activités syndicales. Mais étant donné l'évolution rapide que subit le Québec, il faut être prêt à toute éventualité» ${ }^{28}$.

24 L'année d'internat n'y est pas reconnue puisqu'il y a très peu d'écart entre le salaire accordé à une diététiste sans internat et une diététiste professionnelle. De plus, le salaire des internes doit être négocié à la pièce, tout comme celui des diététistes détenant une maîtrise. «Rapport du Comité des salaires», Rapport annuel, 1970-1971, 14.

25 «President's Report», JCDA, 11,2 (septembre 1949): 46.

26 Voir à ce sujet "Comité des relations professionnelles", $C D Q$ - Rapport annuel, 1965-1966, 8; voir également, «President's Report, 1959-1960», QDA - Annual Report, 1959$1960,4$.

27 «Comité des relations professionnelles», Rapport Annuel, CDQ, 1965-1966, 8.

28 «Rapport de la présidente», $C D Q$ - Rapport annuel, 1965-1966, 13. L'année suivante, la CDQ adhère au Conseil interprofessionnel du Québec, un organisme regroupant une vingtaine de corporations professionnelles et qui reconnaît la pertinence, lors de sa première réunion, de 
Cette avenue s'avère inévitable, à l'aube des années 1970, lorsque l'État refuse de négocier avec les corporations professionnelles toutes les clauses concernant les conditions de travail et que les grandes centrales syndicales - la CSN et la FTQ - déposent des requêtes en accréditation pour l'ensemble des hôpitaux québécois ${ }^{29}$. La citation suivante illustre bien à quel point l'évolution des relations de travail dans le domaine de la santé «condamne» les diététistes à opter pour la voie syndicale:

[...] les groupes des milieux hospitaliers sont dans des situations d'inégalité les uns vis-à-vis des autres: dualité symbolique qui permet, au sein d'un même ministère gouvernemental, des traitements plus favorables à certaines privilégiées. Celles-ci ont eu un jour le malheur d'avoir à se syndiquer. Le geste s'est avéré par la suite heureux et largement récompensé. Voilà qui donne du matériel à réflexion. N'y aurait-il pas pour nous avantage à former un syndicat professionnel plutôt que de continuer vaillamment une lutte inégale avec le fonctionnarisme têtu que l'on connaît? $?^{30}$

La majorité des diététistes opteront alors pour un syndicat indépendant: le Syndicat professionnel des diététistes du Québec (SPDQ), qui sera mis sur pied en $1970^{31}$. Dans un article expliquant les raisons qui ont incité les membres à favoriser ce type de regroupement, la vice-présidente du nouveau syndicat explique que «le SPDQ, en étant composé exclusivement de diététistes protège leur statut et à cause de la scolarité, a une solide base de négociation ${ }^{32}$. Acculées à la syndicalisation, les diététistes choisissent donc de défendre leurs dossiers sur la base de leurs intérêts spécifiques.

\section{LES PHYSIOTHÉRAPEUTES}

L'examen des archives des associations canadienne et québécoise regroupant les physiothérapeutes révèle que ces professionnelles vont suivre un itinéraire similaire. La question du régime de travail semble

«la formule syndicale dans les négociations des conditions de travail». Voir à ce sujet «Assemblée générale du Conseil interprofessionnel du Québec», Bulletin de la $C D Q, 8,1$ (février 1967): 6.

29 Christiane Gauthier-Tougas, «Pourquoi choisir le Syndicat professionnel des diététistes du Québec?», Bulletin de la $C D Q, 2,1$ (mars 1971): 9. 20.

30 Aline Pageau-Lauzière, «Épitre annuel», Bulletin de la $C D Q, 9,3$ (décembre 1969):

31 Ce dernier signera, en 1972, une première convention collective de travail. Cette convention, rétroactive à l'année 1970, sera conclue à la suite des négociations menées par le Cartel des organismes professionnels de la santé (COPS). Voir à ce sujet le Bulletin de la $C D Q$, 4,4 (avril 1973): 26-27.

32 Christine Gauthier-Tougas, op.cit. 
faire surface en 1949. L'Association canadienne de physiothérapie (ACP) souligne en effet, dans les pages de sa revue, que la pénurie permanente de physiothérapeutes est largement due aux bas salaires payés dans les institutions hospitalières où travaille la majorité de ses membres $^{33}$. On rapporte même que plusieurs préfèrent s'exiler aux États-Unis, les salaires y étant plus élevés ${ }^{34}$.

C'est vraisemblablement pour cette raison qu'à partir de cette époque, l'ACP élabore chaque année une «Échelle des salaires recommandés». Ces échelles témoignent d'une nette volonté d'augmenter la rémunération des physiothérapeutes et ce, sur la base des diplômes, de l'expérience acquise et du degré des responsabilités que les physiothérapeutes doivent assumer selon la taille du département et le nombre de personnes qu'elles supervisent. Une description précise des tâches à accomplir pour chacun des postes complète, à l'occasion, ces échelles qui ne cesseront de se complexifier parallèlement à l'expansion des départements de physiothérapie ${ }^{35}$. En 1961, l'ACP recommande par ailleurs que le plafonnement salarial passe de trois à cinq ans. Depuis 1959, l'ACP demandait également d'offrir un supplément salarial à celles qui détenaient une formation plus avancée.

L'ACP ne semble cependant pas jouir, elle non plus, du pouvoir ou de l'influence nécessaires pour imposer ses vues. Bien que ses échelles salariales soient distribuées à l'ensemble des institutions où travaillent les physiothérapeutes, ces dernières doivent négocier leur salaire individuellement, ou encore par l'entremise de la responsable de leur département. Une enquête réalisée à l'échelle nationale en 1964 par l'ACP révèle que ses recommandations salariales sont loin d'être suivies par les institutions hospitalières. On constate qu'en ce qui concerne le Québec, seulement 11 hôpitaux sur les 93 recensés paient les physiothérapeutes en fonction de l'échelle de $1963(11,8 \%)$ et 44 le font selon l'échelle de $1962(46,9 \%)$. C'est donc dire que près de $40 \%$ des hôpitaux recensés paient des salaires inférieurs à ceux recommandés par l'ACP deux ans plus tôt ${ }^{36}$. Ces pourcentages se situent en deçà de la moyenne nationale puisque $19,9 \%$ des hôpitaux canadiens recensés paient des salaires en fonction des barêmes établis

33 «Editorial», Journal of the Canadian Physiotherapy Association (JCPA), 2,7 (décembre 1949): 5 .

34 JCPA, 2,5 (juin 1949): 16.

35 La grille des salaires élaborée en 1948 compte ainsi trois types de poste, soit celui d'assistante physiothérapeute, de physiothérapeute en charge d'un petit département et de chef physiothérapeute (pour les établissements de plus grande taille). Elle comptera six catégories en 1961. Ces informations sont tirées du JCPA. 179.

36 «Rapports annuels des comités permanents - Salaires», JCPA, 16,3 (septembre 1964): 
en 1963. Une nouvelle enquête, réalisée en 1965, démontre que la situation n'évolue guère: les hôpitaux québécois paient, en moyenne, un salaire équivalent à l'échelle recommandée en $1962^{37}$.

Tout au long des années 1960, l'association regroupant les physiothérapeutes québécoises, les Physiothérapeutes de la Province de Québec inc. (PPQ), poursuit pour sa part des négociations, d'abord avec les institutions hospitalières, puis avec le ministère de la Santé afin d'améliorer le régime de travail de ses membres. En 1966, par exemple, elle demande que le salaire initial d'une physiothérapeute diplômée soit établi à 5000 \$ et celui des responsables des départements, entre $7800 \$$ et $8400 \$$ (selon la taille de l'unité de soins). La PPQ revendique également l'application uniforme de cette échelle à travers le Québec, des augmentations annuelles (8 échelons), ainsi que des primes salariales pour qui détient une formation supplémentaire ${ }^{38}$. Mais en 1967, le comité de direction de la PPQ souligne la persistance de plusieurs problèmes: les salaires varient énormément d'un hôpital à l'autre, le niveau de responsabilité n'est toujours pas pris en compte dans la fixation des salaires pas plus, d'ailleurs, que l'expérience acquise $^{39}$. Une enquête menée en 1970 auprès des membres révèle, par ailleurs, que les postes occupés par les assistantes et les physiothérapeutes «seniors» sont mal rémunérés, que la moitié seulement des institutions paie le temps et demi pour les heures supplémentaires, que le travail à temps partiel, assez répandu déjà au sein de cette profession, est généralement sous-payé et que très peu d'institutions reconnaissent encore les études avancées, bien qu'une prime annuelle de $500 \$$ soit assez souvent octroyée aux physiothérapeutes détenant un baccalauréat (plutôt qu'un diplôme) ${ }^{40}$.

Tout comme les diététistes, les physiothérapeutes semblent éprouver des difficultés à faire reconnaître leurs compétences professionnelles par un régime salarial adéquat. Après deux décennies de

37 La situation des physiothérapeutes responsables des départements s'est cependant améliorée, ces dernières étant payées selon les barèmes établis en 1963. Voir le «Rapport du Comité des salaires», JCPA, 17,2 (juin 1965): 99-100. Deux autres enquêtes, menées en 1968 et 1969, indiquent par ailleurs que le Québec se situe toujours bon dernier, avec les provinces maritimes, au chapitre salarial. Voir JCPA, 20,4 (septembre 1968): 280-281, de même que le 21,4 (septembre 1969): 240-241.

$38 \mathrm{PPQ}$, Salaires et conditions de travail recommandés en vigueur rétroactivement le premier juillet 1966.

39 «Procès-verbal de l'Assemblée du Comité de direction des Physiothérapeutes de la Province de Québec inc, 29/11/1967», Minute Books - Stock Ledger - Transfer Book (19611968), n.p.

40 "Procès-verbal de l'Assemblée des membres du Conseil d'administration des Physiothérapeutes de la Province de Québec inc., 09/01/1970», Registre des procès-verbaux et du Capital-actions (1968-1971), n.p. Avant l'année 1966 pour l'Université McGill et 1969 pour l'Université de Montréal, les étudiantes n'obtenaient qu'un diplôme d'études universitaires. 
réticences ${ }^{41}$, ce groupe de professionnelles optera également pour la voie syndicale; le Syndicat professionnel des physiothérapeutes du Québec sera mis sur pied en $1970^{42}$. Le président du nouveau syndicat rappelle que les négociations se font maintenant à l'échelle provinciale et que l'État refuse désormais de «négocier les conditions de travail avec des corporations professionnelles et ne veut avoir affaire qu'aux syndicats» ${ }^{43}$. Il ajoute, sur un ton qui semble résigné: "pour ne pas nous retrouver encore une fois sous-représentés et passer inaperçus dans les prochaines négociations (...) il a fallu recourir au moyen qui, de toute évidence, nous donnerait des chances égales au départ: le syndicalisme. ${ }^{44}$

La rationalisation de la gestion de la main-d'œuvre entreprise par l'État oblige ainsi les physiothérapeutes à se doter d'outils plus efficaces pour négocier les conditions d'exercice de leur pratique et, notamment, le régime de travail auquel elles sont soumises. Elles se regrouperont toutefois sur une base autonome privilégiant, tout comme les diététistes, un syndicat homogène au sein duquel leurs revendications pourront acquérir davantage de poids.

\section{LES TECHNOLOGISTES}

L'amélioration du régime de travail préoccupera également l'Association canadienne des technologistes de laboratoire (ACTL). Ainsi, un article rappelant les progrès accomplis par l'ACTL depuis sa fondation souligne, entre autres, que l'association a multiplié les efforts, non sans succès, pour améliorer le régime salarial de ses membres $^{45}$. Au début des années 1950, l'ACTL publie une échelle des salaires recommandés afin d'obtenir pour ses membres un salaire «raisonnable» ${ }^{46}$.

41 Voir notamment à ce sujet «A Tradition and a Challenge - A Message from our Honorary President, Mr Vincent Price», JCPA, 10,3 (septembre 1958): 132. Voir également «Nouvelles de l'exécutif national» dans le 17,1 (janvier 1965): 9. Ces deux articles témoignent de façon évidente du refus de l'ACP de voir ses membres se syndiquer, notamment parce que la physiothérapie est un service essentiel à la population et que la grève est inconciliable avec l'éthique de la profession. COPS.

42 Il signera une première convention collective en 1972 négociée également par le 241.

43 Yvan Harvey, «Le syndicalisme en physiothérapie», JCPA, 22,5 (décembre 1970):

44 Ibid.

45 Canadian Journal of Medical Technologist (CJMT), 10 (1948): 136-137.

46 Nous n'avons pu retrouver cette échelle salariale. Cette information est tirée d'un article de J. Chamberlin et V. Booth paru dans le numéro spécial du CJMT publié à l'occasion de $50^{\circ}$ anniversaire de la fondation de l'ACTL et intitulé, «Les décennies», 20. 
Dès 1955, les technologistes du Québec se dotent par ailleurs d'une association syndicale, l'Association des diplômés en technologie médicale (ADTM). Ce regroupement entend non seulement défendre les intérêts économiques de ses membres, mais aussi leurs intérêts corporatifs ${ }^{47}$. Au début des années 1960, l'ADTM accorde néanmoins une attention plus particulière à l'amélioration des conditions de travail à la suite, notamment, de nombreuses pressions de sa «base (...) qui exprimait de plus en plus son insatisfaction des salaires et des conditions de travail auxquels elle était soumise» ${ }^{48}$. L'ADTM élabore alors une première échelle salariale et la soumet à l'Association patronale des services hospitaliers (APSH) de Québec. On y décèle une nette volonté d'établir la rémunération en fonction de la formation reçue, des années d'expérience et du niveau de responsabilité assumée. L'échelle de salaire proposée par l'ADTM prévoit ainsi cinq types de poste et une progression salariale échelonnée sur douze années ${ }^{49}$. Elle demande également qu'un supplément salarial annuel de $500 \$$ soit alloué aux technologistes détenant une spécialisation $^{50}$. Les clauses du premier mémoire d'entente que conclut l'ADTM avec l'APSH de Québec en 1960 se situent cependant en deçà de ces demandes. Les échelles de salaires ne sont établies qu'en fonction des années d'expérience (jusqu'à concurrence de dix) et la spécialisation ne confère à ses détenteurs qu'un supplément salarial de $5 \$$ par semaine ${ }^{51}$. Une deuxième entente est signée en 1962 mais,

47 L'ADTM entame ainsi des démarches auprès des autorités gouvernementales afin que la profession soit légalement reconnue. Jusqu'en 1965, elle entretient par ailleurs des espoirs de fusion avec la section québécoise de l'ACTL, section qui deviendra, en 1964, l'Association des technologistes médicaux du Québec et, en 1967, la Corporation des technologistes médicaux du Québec. C'est cette dernière qui sera habilitée à regrouper les technologistes en chambre professionnelle et l'ADTM devra alors restreindre ses activités à la négociation des conditions de travail de ses membres. Voir à ce sujet Nicole Bélanger, Le développement des organisations syndicales et corporatives des techniciens en laboratoire au Québec, thèse de M.A. (sociologie), Université du Québec à Montréal, 1978, 230 p. Voir aussi N. Bouffard, La technologie médicale au Québec (APTMQ, 1975).

48 N. Bélanger, op. cit., 127.

49 Il y a trois catégories pour les technologistes ne détenant aucune responsabilité. Les deux autres catégories sont celles de technologistes en charge de sections de laboratoire et de chefs technologistes responsables de l'ensemble du laboratoire.

50 Les technologistes peuvent acquérir une formation générale puis opter pour une des spécialisations offertes comme la bactériologie, la biochimie, la cytologie, l'hématologie, l'histologie, la pathologie, etc. «Échelle de salaire proposée par l'ADTM - 1960», ADTM APTMQ - Procès-verbaux, 1955-1966 - Correspondance, 1955-1964, section «Travail», n.p.

51 «Mémoire d'entente entre l'ADTM et l'APSH. Clauses salariales applicables du 01/ 04/1960 au 01/04/1962», Ibid. Ce contrat de travail ne s'applique vraisemblablement qu'à quelques hôpitaux de la région de Québec. 
mises à part les augmentations salariales, l'ADTM ne fera aucun gain $^{52}$.

Il faut spécifier ici que l'ADTM ne dispose que d'une faible marge de manœuvre dans ses négociations avec l'APSH puisqu'elle n'a jamais demandé d'accréditation syndicale. Elle ne peut donc que soumettre des recommandations et en discuter avec les autorités hospitalières. Malgré sa charte syndicale, l'ADTM se retrouve donc, en quelque sorte, dans la même position que les associations de diététique et de physiothérapie, ne disposant que d'un simple pouvoir de représentation ${ }^{53}$.

À partir de 1965, l'ADTM entame toutefois une véritable campagne d'organisation syndicale. Elle change également d'appellation, devenant l'Association professionnelle des technologistes médicaux du Québec (APTMQ). À la fin de 1968, une vingtaine d'ententes sont conclues avec autant d'hôpitaux ${ }^{54}$. L'année suivante, l'APTMQ signe une première convention collective de travail négociée à l'échelle provinciale. Elle obtient des gains plus qu'appréciables pour ses 820 membres cotisants, non seulement au niveau salarial, mais aussi en qui concerne le régime de travail dans son ensemble, la classification du personnel, etc. Le salaire de base du personnel débutant est alors fixé à $95 \$$ par semaine, ce qui constitue une hausse moyenne de plus de $15 \%$.

Il semble bien que l'évolution des relations de travail dans le secteur hospitalier ait influencé également l'action de l'APTMQ. À la

52 «Convention collective professionnelle entre l'ADTM et l'APSH». Détail intéressant, les échelles de salaire accordées en 1960 et 1962 présentent une double structuration, une pour les femmes et une autre pour leurs confrères. Le salaire de ces derniers est de dix dollars de plus par semaine et ce, dans toutes les catégories d'emploi. Bien que les sources soient muettes à ce chapitre, on sait que ces pratiques discriminatoires envers le personnel féminin furent longtemps entérinées par plusieurs regroupements syndicaux. En ce qui concerne le milieu hospitalier, voir D. Gaucher, Le maternage mal salarié - Travail sexué et discrimination salariale en milieu hospitalier (Montréal, Presses de l'Université de Montréal, 1983). Voir aussi Nadia Fahmy-Eid et Lucie Piché, Si le travail m'était conté... autrement. Les travailleuses de la CTCC-CSN: quelques fragments d' histoire, 1921-1976 (Montréal, CSN, 1987).

53 Une première acccréditation lui sera octroyée en 1961 pour le personnel féminin des laboratoires de l'Université Laval. Leurs confrères ne les rejoindront qu'en 1965, étant déjà syndiqués à la CSN.

54 L'ADTM cherchera dès lors à regrouper l'ensemble des technologistes du Québec. Une partie de cette main-d'œuvre, déjà syndiquée au sein de la Fédération nationale des services (FNS-CSN), hésitera cependant à changer d'allégeance syndicale, la FNS étant beaucoup plus combative. C'est, du moins, ce qu'affirme N. Bélanger, op. cit., 168-169. Il est cependant vrai que l'ADTM fut longtemps assise entre deux chaises, hésitant à assumer pleinement son rôle syndical. Voir également à ce sujet N. Bouffard, op. cit.

55 J. Lafrenière, «Hôpitaux du Québec. Signature d'un premier contrat de travail», La Presse, 13 août 1969; reproduit dans Techno-info, 5,4: 7. Cette convention est rétroactive au ler juillet 1968 et s'applique jusqu'au 30 juin 1971. 
suite de négociations infructueuses avec le Comité mixte des relations du personnel de l'Assurance hospitalisation en 1965, elle affirme en effet que pour «suivre la politique actuelle du Gouvernement, la production de notre charte syndicale s'avérait indispensable pour que nous puissions exposer officiellement nos revendications ${ }^{56}$. L'APTMQ refusera cependant de compromettre ses relations avec l'État et se désolidarisera des mouvements de grève qui marquent alors le secteur de la santés7. Précisons enfin que la Corporation des technologistes médicaux (CTMQ) jouera, quant à elle, un rôle plus effacé en ce qui concerne l'amélioration du régime de travail auquel sont soumises les technologistes. On peut présumer que l'existence de l'ADTM-APTMQ la décharge de cette préoccupation, d'autant plus que des liens très étroits existent entre ces deux regroupements.

Ainsi, mises sur pied afin de favoriser le développement de leurs professions respectives, les association canadiennes et québécoises de diététique, de physiothérapie et de technologie médicale s'appliqueront à faire reconnaître les compétences de leurs membres par un régime de travail adéquat. Elles le feront en tablant notamment sur la formation de leurs membres: une formation de niveau universitaire, qui comporte un apprentissage de connaissances spécialisées et qui conduit ces praticiennes à poser des actes à caractère professionnel. Ces arguments également évoqués, on le verra, pour obtenir un statut professionnel légal, ne semblent toutefois pas suffisants pour doter ces associations d'un pouvoir de négociation leur permettant d'arriver à leurs fins. L'intervention étatique dans le champ de la santé incitera toutefois ces travailleuses à s'engager dans une politique syndicale, ce qui leur donnera des moyens accrus pour faire valoir leurs revendications. Devenu employeur d'une main-d'œuvre dont les effectifs augmentent à une cadence accélérée, l'État cherchera, de son côté, à rentabiliser les coûts de cette main-d'œuvre ce qui, paradoxalement, aura un impact positif pour les travailleuses de la santé. Le roulement élevé du personnel hospitalier risque, en effet, de miner l'efficacité du système que l'État tente de mettre en place. À titre d'exemple, jusqu'au milieu des années 1960, la durée moyenne de la carrière d'une technologiste est de 2,1 ans, celle d'une diététiste de 5 ans et seulement $25 \%$ des physiothérapeutes pratiquent encore après 5 ans $^{58}$. Le fait que la structure d'emploi du monde hospitalier repose largement sur l'embauche d'un personnel féminin explique en partie ce

56 Techno-Info, 2,1 (janvier 1966).

57 Techno-Info, 5,6 (1969). Cité par Nicole Bélanger, op. cit., 166.

58 B. L. P. Brosseau, m.d., «Problèmes de main-d'œuvre», L'hôpital d'aujourd' hui, 13,11 (novembre 1967): 16-17. 
phénomène puisque le mariage «décime» continuellement les rangs de plusieurs de ces professions. Au début des années 1960, de plus en plus d'intervenants s'accordent cependant pour reconnaître que des salaires plus élevés retiendraient davantage les femmes sur le marché du travail et les inciteraient à y retourner après leur mariage. L'amélioration du régime de travail risque par ailleurs d'attirer davantage les hommes, stabilisant ainsi la profession ${ }^{59}$. En 1962, la Commission d'enquête sur l'assurance-santé (Commission Hall), prône de telles mesures. Quelques années plus tard, le directeur de l'Institut supérieur d'administration hospitalière de l'Université de Montréal, G. Blain, reprend à son compte ces recommandations:

Il est à espérer que, grâce à ces mesures, un plus grand nombre d'hommes, dans l'avenir, s'orienteront vers certaines professions paramédicales, comme par exemple, la diététique, la physiothérapie, la technologie médicale et même le nursing (...) Le coût du relèvement des salaires suggérés par le Rapport Hall sera amplement compensé à long terme par les économies assurées par une plus grande stabilité du personnel. ${ }^{60}$

C'est reconnaître, implicitement, que les salaires offerts antérieurement étaient des «salaires de femmes» et que le cheminement de carrière était particulièrement limité. L'intervention de l'État permet donc à ces praticiennes de la santé d'accéder, jusqu'à un certain point, à un régime de travail qui prend davantage en considération les compétences professionnelles qu'elles ont développées. Cette reconnaissance devra cependant être négociée par la voie syndicale et non sur la base de la reconnaissance du seul prestige professionnel.

\section{2 - LES ENJEUX LIÉS À L'OBTENTION D'UN STATUT PROFESSIONNEL LÉGAL DANS L'UNIVERS DE LA SANTÉ}

Les associations de diététique, de physiothérapie et de technologie médicale prennent rapidement conscience que la défense des intérêts de leurs membres déborde le cadre des conditions de travail et passe également par l'obtention d'un statut professionnel légal. Aussi, dès la fin des années 1940, elles entreprennent des démarches pour obtenir un statut de corporation afin de pouvoir ainsi protéger leur titre d'emploi et, surtout, obtenir un droit de pratique exclusif sur un

59 Cette position est également défendue par les associations. Voir notamment à ce sujet Louise Britain, «Where Are We Going?», JCDA, 15,2 (septembre 1953): 11. «La technologie médicale en tant que profession», Techno-info, 3,1 (février 1967): 8-10.

60 «L'éducation permanente et le domaine paramédical», L'hôpital d' aujourd' hui, 13,1 (janvier 1967): 17. 
certain nombre d'actes professionnels. Il importe, en effet, de déterminer avec plus de précision les frontières de la compétence reconnue à chacune des nombreuses professions qui, désormais, affirment toutes assumer une part active dans le processus de guérison.

L'étatisation des soins de santé incite l'État à revoir, de son côté, ses propres politiques en matière de réglementation professionnelle ${ }^{61}$. Auparavant, souligne A. Petitat dans son ouvrage sur les infirmières, «la corporation libérale se faisait attribuer la gestion d'un territoire que l'État n'avait pas l'intention d'administrer ${ }^{62}$. Devenu employeur de plusieurs groupes bénéficiant déjà d'un statut corporatif ou espérant l'acquérir, l'État cherche désormais à modifier les règles du jeu. Les trois professions seront donc confrontées, chacune à leur façon, au pouvoir de régulation du champ socio-sanitaire que détient l'État. Le corps médical, de par les prérogatives qui lui sont reconnues en matière de soin, constituera également un intervenant majeur. L'obtention du statut de corporation fermée confère, rappelons-le, une certaine autonomie dans l'organisation et la gestion du savoir et de la pratique professionnelle; d'où la volonté affirmée du corps médical d'exercer un droit de regard sur les revendications de ces nouvelles intervenantes.

Précisons ici qu'aucune de ces professions ne prétend concurrencer les médecins en ce qui concerne l'établissement d'un diagnostic médical. Toutes font cependant valoir que leurs membres ont acquis un bagage de connaissances spécialisées ou des habiletés techniques leur permettant de dispenser des soins en vue de rétablir la santé des malades - c'est le cas de la diététique et de la physiothérapie - ou encore, comme en technologie médicale, d'aider le médecin à poser un diagnostic ${ }^{63}$. C'est sur la base de ces critères et après avoir uniformisé la formation de leurs membres et établi des normes en ce qui a trait à leur pratique, que ces trois professions vont entamer des démarches légales auprès de l'État pour obtenir un statut de corporation fermée.

61 La réglementation professionnelle est une juridiction provinciale. C'est d'ailleurs en partie pour cette raison que les associations canadiennes inciteront leurs sections locales à obtenir des chartes d'incorporation provinciales.

62 A. Petitat, op. cit., 83.

63 Les codes d'éthique qu'élaborent ces professionnelles sont d'ailleurs très explicites à cet égard. En ce qui concerne la diététique, voir notamment R. Beaudoin, «Statut professionnel dans l'hôpital d'aujourd'hui», L'hôpital d'aujourd' hui, 5,10 (octobre 1959): 51. Voir aussi F. Goulet et C. de Girardi, «De la science et de l'art de la physiothérapie», L'hôpital d'aujourd' hui, 14,9 (septembre 1968): 55. Pour la technologie médicale, voir la définition de la convention collective de travail signée en 1962 entre l'ADTM et l'Université Laval, ADTM APTMQ - Procès-verbaux, 1955-1966 - Correspondance, 1955-1965, n.p. 
En 1956, l'Association québécoise de diététique présente son projet d'incorporation devant la législature du Québec et obtient en partie gain de cause. Le statut de corporation lui est octroyé et seuls les membres dûment inscrits auprès de la corporation peuvent désormais porter le titre de diététistes ${ }^{64}$. Ce monopole n'est cependant pas assorti d'un droit de pratique exclusif. La corporation présentera sans succès un nouveau projet de loi à cet effet en 1966, puis en 1972, lors des audiences de la commission chargée de l'élaboration du code des professions ${ }^{65}$.

La nouvelle corporation se voit néanmoins conférer, outre l'inscription des membres, l'élaboration et la supervision des programmes d'études universitaires, la mise en place et la surveillance des internats, la discipline des membres, etc. Cette loi oblige également les institutions hospitalières à «confier l'entière direction des départements de diététique à des diététistes professionnelles» ${ }^{66}$. Celles-ci supervisent l'ensemble de la gestion de la cuisine hospitalière - de l'achat de la nourriture à la distribution des repas. À ces fonctions administratives s'ajoutent des tâches liées à la recherche, de même que l'enseignement aux clientèles étudiantes de diététique, de sciences infirmières et de médecine.

L'étatisation du système de santé menace toutefois ces acquis. Les règlements adoptés en 1969 par le gouvernement pour faire suite à la Loi des hôpitaux de 1962, évincent les diététistes du personnel de direction des hôpitaux et confient la gestion des départements à un personnel ayant acquis une formation en administration hospitalière et qui relève dorénavant des services auxiliaires. La diétothérapie, l'enseignement et la recherche passent pour leur part sous la responsabilité du directeur médical. Devenu le principal bailleur de fonds, l'État entend s'assurer ainsi que la gestion des coûts de la cuisine hospitalière soit assumée par des gestionnaires (souvent masculins d'ailleurs) formés spécifiquement à cet $\operatorname{effet}^{67}$. La CDQ réagira

64 Loi des diététistes, 4-5 Elizabeth II, chap. 156, 1956.

65 Cette dernière information est tirée de C. A. Sheppard, L'organisation et la réglementation des professions de la santé et du bien-être social au Québec, Annexe 12, tome 2. Document produit pour la Commission d'enquête sur la santé et le bien-être social au Québec (Québec, n.d.), 596.

66 «Recommandations concernant les règlements adoptés en vertu de la Loi des hôpitaux - présentées au ministère de la Santé par la Corporation des diététistes du Québec, Montréal, mai 1969», JCDA, 31,2 (juin 1970): 90.

67 Ibid. Cette tentative d'éviction des diététistes de la direction hospitalière rejoint d'une certaine façon, les propos de N. Laurin et D. Juteau qui affirment que «le transfert de ces services à l'État entraînera alors la mise au rancart et éventuellement la disparition des religieuses, qui seront remplacées par des travailleuses salariées dans la plupart des postes de travail sauf dans les postes de direction et d'encadrement des services dont les hommes, fonctionnaires de l'État, 
vivement à cette nouvelle réorientation, affirmant qu'une telle réorganisation est contraire à la Loi des diététistes de 1956. Elle exercera de nombreuses pressions auprès du ministère de la Santé, de même qu'auprès de la direction des hôpitaux afin «de réintégrer le diététiste directeur au même niveau que les autres directeurs ${ }^{68}$. Elle obtiendra finalement gain de cause en 1973, à la suite de l'adoption de la Loi 65.

Cette victoire des diététistes a son importance; elle témoigne d'une lutte constante pour établir la crédibilité de leur travail et le faire accéder au rang de profession. Depuis le tournant du siècle, les diététistes ont en effet été formées, du côté des francophones, dans les Écoles ménagères, alors que les «Schools of Household Sciences» ont dispensé la formation aux anglophones. Ces programmes, mis sur pied à l'origine pour procurer aux filles une formation leur permettant de remplir «scientifiquement» leur futur rôle de ménagère, offrent bientôt, à la faveur du développement des connaissances en nutrition, une spécialisation en diététique ${ }^{69}$. Recrutées par les hôpitaux pour élaborer et superviser les diètes (diétothérapie), les diététistes mettent bientôt sur pied des départements et assument la gestion de l'ensemble de la cuisine hospitalière - un acquis que la loi de 1956 viendra entériner et que les «Règlements» de 1969 mettront en péril.

Si les diététistes réussissent à se maintenir au sommet de la direction de leur département, elles semblent cependant éprouver des difficultés à s'intégrer à l'équipe de soins. Leur malaise à cet égard semble trouver sa source dans la non reconnaissance, par les médecins, de leurs compétences spécifiques. Depuis longtemps dénoncée ${ }^{70}$, cette attitude du corps médical à l'endroit des diététistes risque d'avoir des conséquences particulièrement lourdes au cours des années 1960 alors que s'élabore une nouvelle vision du travail sanitaire et qu'émerge la notion d'équipe de soins. Une diététiste résume ainsi la situation:

s'empareront». "L'évolution de l'appropriation des femmes: des religieuses aux «mères porteuses»", Revue canadienne de sociologie et d'anthropologie, 25,2 (1988): 198.

68 «Rapport du comité du statut du diététiste dans les hôpitaux», Rapport annuel - 19701971, 19. L'emploi du masculin commence à se répandre à cette époque ce qui dénote peut-être une volonté de contrer l'image de profession féminine qui est accolée à la diététique.

69 La science de la nutrition se développe à la suite des progrès réalisés en chimie organique.

70 En témoignent, ces propos publiés en 1940: "contrary to the general belief that a dietician is a glorified cook, a dietitian plays an important role in the scientific nutrition field which controls the health of the nation». «Editorial - What is a Dietitian», JCDA, 2,1 (juin 1940): 16. 


\begin{abstract}
Demandez-lui (au médecin) le rôle de la diététicienne à l'hôpital, et il vous répondra qu'elle est là pour enseigner les régimes. Mais est-ce là tout ce qu'elle peut apporter? On oublie qu'elle est une spécialiste (...) en ce qui concerne l'alimentation (...) On l'oublie même au point qu'on ne la juge pas apte à réfuter certaines diètes erronées, basées sur des conceptions subjectives et arbitraires, et non sur des principes scientifiques. ${ }^{71}$
\end{abstract}

On peut se demander, par ailleurs, si le fait que le savoir et la pratique de la diététique ne se situent pas d'emblée dans le champ médical ne permet pas aux diététistes d'échapper au contrôle immédiat des disciples d'Esculape. Ainsi, lorsque ces professionnelles présentent leur projet d'incorporation auprès de l'État en 1956, les médecins ne semblent pas intervenir; bien qu'elles n'obtiennent pas, on l'a vu, l'exclusivité de la pratique, elles acquièrent tout de même assez facilement un statut de corporation. Le libéralisme de l'État à cette période (avant 1960) y est sûrement pour quelque chose. Mais il reste que la non-intervention du corps médical est surprenante. La situation vécue en physiothérapie et en technologie médicale le confirme.

En 1965, l'association regroupant les physiothérapeutes du Québec (PPQ), présente en effet devant la législature un projet de loi afin d'obtenir le monopole du titre et du champ d'exercice ${ }^{72}$. Ce projet, qui tombe dans l'oubli avec l'arrivée d'un nouveau gouvernement en 1966, est par la suite ajourné jusqu'à ce que la réorganisation complète du système scolaire et celui des soins de santé soit complétée. Ce n'est qu'en 1973, lors de la réforme du Code des professions, que les physiothérapeutes obtiennent une reconnaissance légale. Dans leur cas également, la protection de leur titre leur est accordée, mais non le monopole de la pratique.

Durant la période comprise entre le dépôt du premier projet de loi et l'obtention du statut de corporation professionnelle, les physiothérapeutes doivent cependant s'engager dans une vive bataille contre le processus de déqualification qui menace leurs aspirations professionnelles. Le coup d'envoi est donné par le Comité d'étude sur les professions auxiliaires de la santé (Comité Bergeron). Ce comité, mis sur pied par les ministères de la Santé et de l'Éducation, est composé

71 S. Mavrikakis, L'hôpital d' aujourd' hui, 15,1 (janvier 1969): 15. Une autre diététiste se plaint pour sa part de l'attitude des «docteurs» (qui) varie d'un hôpital à l'autre. Certains établissements présentent une équipe médicale dynamique qui croit à la diététique et aux diététiciennes et y font appel pour des calculs spéciaux, les invitent aux conférences, aux études de cas, aux tournées médicales (...) Cette situation, quoique très possible, demeure exceptionnelle». L. Mercier, «À qui la faute?», L'hôpital d'aujourd' hui, 15,10 (octobre 1969): 50.

72 Les Physiothérapeutes de la Province de Québec Inc, Private Bill no 140 - An Act to Incorporate the Quebec Society of Physiotherapists, avril 1965. 
presque exclusivement de médecins. Il a pour mandat d'identifier les professions auxiliaires de la santé, de préciser leur champ respectif d'exercice et d'émettre des recommandations en ce qui a trait à leur formation.

Les physiothérapeutes sont alors classées dans la catégorie du personnel technique et leurs fonctions, décrites comme étant «l'application de certains traitements par agents physiques ${ }^{73}$. Le rapport Bergeron recommande que la formation des emplois présentant un caractère technique soit reléguée au niveau collégial. L'association des physiothérapeutes avait pourtant fait valoir, dans le mémoire qu'elle avait soumis à ce comité, qu'un niveau universitaire s'imposait puisque «le physiothérapeute est responsable de tous les traitements en physiothérapie, depuis l'évaluation, le choix et l'exécution des modalités, la discussion avec le médecin traitant, la réévaluation en cours de traitement $(. ..) \gg^{74}$.

Si l'association ne réussit pas à empêcher la création d'un cours de niveau collégial - il sera offert à partir de 1969 et conférera un DEC en technique de réadaptation - la formation universitaire n'est pas éliminée pour autant. Les recommandations d'un Comité ad hoc, formé de représentants des ministères de l'Éducation, des Affaires sociales, du réseau collégial et universitaire, et mandaté pour évaluer le mode d'intégration des futurs diplômés du CEGEP, viennent cependant raviver le débat. Ce comité recommande en effet que le personnel formé par le réseau collégial soit considéré, sur le marché du travail, sur le même pied que les physiothérapeutes ayant acquis leur formation à l'université. Il allègue à cet égard que les techniciennes sont habilitées à effectuer $95 \%$ des tâches dévolues aux physiothérapeutes et que ce pourcentage pourrait, en réajustant quelque peu le contenu du cours, être haussé à $100 \%$. Dans l'esprit du comité, le baccalauréat en physiothérapie devrait être orienté uniquement vers une formation en administration et en pédagogie. La logique qui sous-tend ce choix est très explicite puisque le comité affirme qu'il «est difficile de concilier un certain degré d'autonomie professionnelle qui est rattaché à ce niveau de formation (universitaire) avec une intégration efficace dans une équipe cohérente de soins ${ }^{75}$. Avec la réorganisation du

73 «Inventaire des professions auxiliaires de la santé de la Province de Québec», Annexe I, Rapport du Comité des professions auxiliaires de la santé sur la première partie de son mandat au lieutenant-gouverneur en conseil - Rapport préliminaire, n.p.

74 Mémoire de "Les Physiothérapeutes de la Province de Québec Inc.» au Comité d'étude des professions auxiliaires de la santé, 1968, 8.

75 Rapport du Comité ad hoc sur l'intégration des techniciens en réadaptation au marché du travail, 1972, 19. 
système de santé, on semble en effet vouloir consolider l'organisation pyramidale de la division du travail sanitaire et, conséquemment, tendre vers l'intégration de techniciens plutôt que de professionnels autonomes.

Lorsque les physiothérapeutes présentent un nouveau projet de loi lors des audiences de la commission parlementaire mandatée pour élaborer le Code des professions, l'Association des physiâtres du Québec intervient à son tour pour contrer les revendications des physiothérapeutes. Ces spécialistes de la médecine physique soutiennent alors que «le physiothérapeute reçoit une formation technique qui lui permet simplement d'appliquer un traitement prescrit par un médecin. Il n'est aucunement qualifié pour assumer la responsabilité du traitement» ${ }^{76}$. La position défendue par le Collège des médecins et chirurgiens du Québec est identique ${ }^{77}$.

La réorganisation du système de réglementation professionnelle exacerbe, en fait, des tensions qui perdurent depuis plus d'une décennie. Il faut voir, en effet, que dans la première moitié du $\mathrm{XX}^{\mathrm{e}}$ siècle, la médecine physique et la physiothérapie sont à peu près inconnues dans le champ de la santé. En 1955, le Canada ne compte que 18 spécialistes en médecine physique ${ }^{78}$. Une des conséquences de cette rareté est que les physiothérapeutes travaillent souvent seules, traitant certes des patients référés par des médecins, mais dont elles assument l'entière responsabilité du traitement. L'accroissement du nombre de spécialistes en médecine physique vient bouleverser cette dynamique dans la mesure où les physiâtres tendent à investir le processus de soins en établissant avec précision le plan de traitement, la nature exacte des soins à dispenser, etc. Les physiothérapeutes risquent ainsi de se voir reléguées à un rôle de simples exécutantes ${ }^{79}$. Elles réagissent alors vivement, affirmant que leur formation leur permet d'établir la nature des traitements, de procéder à la réévaluation constante de la condition du patient, etc. Au cœur du débat, c'est bien de l'autonomie professionnelle des physiothérapeutes qu'il s'agit, c'est-à-dire de la nature des actes qu'elles peuvent poser et de leur capacité de dispenser des soins et non pas simplement «d'appliquer»

76 Mémoire présenté à la Commission parlementaire en relation avec le Bill 272 proposant une législation gouvernant l'exercice de la physiothérapie, 11 février 1972, 8-9.

77 Mémoire du Collège des médecins et chirurgiens de la Province de Québec à la Commission parlementaire spéciale sur les corporations professionnelles, février 1972.

78 «Constructive Criticism of Occupational and Physical Therapy», JCPA, 7,2 (septembre 1955): 7.

79 Ce phénomène est par ailleurs amplifié par l'introduction d'appareils de traitement toujours plus complexes qui remplacent, en partie du moins, les thérapies manuelles. 
un traitement entièrement pré-établi. En raison de la formation des physiothérapeutes, il importe, soutient-on, d'obtenir:

more freedom of action than we appear to have at present. We would suggest that we should treat patients only with «medical referral», that is, not directions; and that the therapists themselves, «after carefull evaluation», plan, implement and if necessary, change their own treatment regimes (...) We should seem to be at the cross-roads. Are we to continue in our increasingly subsidiary role or are we going to develop into a truly respected profession with all that this implies?

Les physiothérapeutes doivent donc affronter à la fois les médecins et l'État qui, pour des raisons forts différentes, tentent de limiter leur autonomie professionnelle. Dans sa volonté de réorganiser le système de santé et de le faire à moindre frais, l'État-employeur tend, en effet, à revenir sur la politique libérale qui caractérisait sa gestion des corporations avant les années 1960. Cette nouvelle orientation rejoint, jusqu'à un certain point, les intérêts des physiâtres.

Le projet de loi que présente l'Association des diplômés en technologie médicale (ADTM) devant la législature provinciale en 1958 soulève, pour sa part, autant l'opposition des médecins que celle des administrations hospitalières. La Loi des technologistes médicaux du Québec prévoit non seulement que seuls les membres inscrits au registre de l'ADTM pourront porter le titre de technologiste de laboratoire, mais également qu'eux seuls pourront effectuer les analyses de laboratoire. C'est bien ce droit de pratique exclusive qui indisposera les médecins. Réunis au lendemain du dépôt de ce projet de loi, ces derniers s'opposent en premier lieu à ce que les aides-technologistes soient exclus du projet de loi. Le travail de ce personnel non spécialisé est d'autant plus précieux, soutiennent les médecins, que les technologistes ne sont pas en nombre suffisant pour soutenir la demande des laboratoires. Il n'est par ailleurs pas du tout prouvé, soutient pour sa part le Conseil des hôpitaux dans un communiqué qu'il fait parvenir aux participants, qu'il est pertinent d'ériger en corporation professionnelle l'ADTM, puisque «le niveau de leurs études [des technologistes] et de leurs responsabilités ne leur donne pas droit à ce privilège ${ }^{81}$. Plus encore, soutient un médecin présent à cette réunion,

80 R. Walmsley et G. Snewing, "The Changing Role of the Physical Therapist in Canada», JCPA, 22,5 (décembre 1970): 250.

81 «Procès-verbal d'une réunion tenue à l'Université de Montréal le 7 janvier 1958, en vue de la discussion du Bill privé no 163 ou Loi des technologistes médicaux du Québec», archives privées (R. Donaldson), n.p. 
le monopole du droit de pratique que demande l'ADTM, «foule au pied les intérêts sacrés des médecins» dans la mesure, explique l'un de ses confrères, où «l'adoption du Bill aurait pour effet d'empêcher un chimiste, biologiste, physicien, etc., même munis du Ph.D., de travailler dans un laboratoire, d'y faire de la recherche, des analyses et d'empêcher les infirmières de faire des prélèvements et analyses banales ${ }^{82}$. Le projet de loi stipule pourtant que le droit d'exercice peut être attribué à d'autres professionnels et qu'il doit être exercé sous contrôle médical ${ }^{83}$. Mais il semble que de telles garanties ne soient pas jugées suffisantes. Ce projet sera finalement rejeté en troisième lecture, à la suite du tollé des divers intervenants de la santé dont les médecins de laboratoire. Ces derniers mettront d'ailleurs sur pied leur propre regroupement en 1959, soit l'Association des médecins de laboratoire ${ }^{84}$.

Le flou juridique dans lequel continuera d'évoluer la profession sera maintes fois dénoncé par l'ADTM puis par la CTMQ qui soutiendra, pour sa part, qu'il lui est impossible d'assurer la qualité des actes professionnels des technologistes puisque aucune norme ne régit l'embauche du personnel et l'organisation du travail. Le fait que les hôpitaux préfêrent engager un personnel non qualifié qu'ils paient en moyenne $15 \%$ moins cher n'est pas étranger à ce problème de qualité, soutient également la corporation devant la Commission Castonguay en $1967^{85}$. Plus du tiers des 2000 personnes travaillant dans les laboratoires ne posséderait pas de formation adéquate et aurait été formé sur le $\operatorname{tas}^{86}$. Tout au long des années 1960, l'ADTM et la CTMQ revendiqueront d'ailleurs non seulement l'emploi d'un personnel qualifié, mais aussi une restructuration complète du programme de formation des technologistes, proposant notamment la mise en place d'un enseignement scientifique plus poussé et d'un diplôme universitaire plus élevé. Ces demandes resteront lettre morte et la technologie médicale devra même quitter, en 1970, le niveau de l'enseignement universitaire. La formation des technologistes sera désormais dispensée dans les CEGEPS, consolidant ainsi un peu plus l'organisation

82 Ibid. Soulignons ici que les associations regroupant les infirmières s'opposeront également à ce projet de loi.

83 «Loi des technologistes médicaux du Québec», reproduite dans l'ouvrage de N. Bouffard, op. cit., 141.

$84 \mathrm{~N}$. Bélanger affirme que la mise sur pied de cette association résulte d'une volonté de court-circuiter toute nouvelle tentative d'incorporation des technologistes. Op. cit., 93-94.

$85 \mathrm{CTMQ}$, Mémoire soumis à la Commission d'enquête sur la santé et le bien-être social - Mémoire no 39, avril 1967, 19.

86 C. Dutrisac, «Devant la Commission Castonguay, la Corporation des technologistes médicaux du Québec s'inquiète de la pénurie de technologistes compétents», La Presse, 27 mars 1968, reproduit dans Techno-Info, 4,2 (mars 1968): n.p. 
pyramidale de la division du travail sanitaire. La CTMQ demandera néanmoins de nouveau la protection de ses titres et le droit exclusif de la pratique lors des audiences de la commission chargée d'élaborer le Code des professions ${ }^{87}$. Invoquant notamment la protection de la santé publique pour légitimer ses demandes, la corporation fera valoir que les médecins et autres professionnels de la santé orientent directement leurs diagnostics et leurs thérapeutiques en fonction des analyses réalisées en laboratoire; d'où l'importance de s'assurer du contrôle de la qualité des actes posés. Le corps médical évaluera, quant à lui, que ce contrôle doit être exercé par les pathologistes, les biochimistes et les autres médecins spécialisés dans le travail de laboratoire et qui assurent déjà la supervision du travail. C'est cette dernière option qui prévaudra lors de la réforme du Code des professions puisque la corporation n'obtiendra que le monopole de son titre.

\section{CONCLUSION}

L'analyse des luttes menées par les associations canadiennes et québécoises de diététique, de physiothérapie et de technologie médicale permet de mieux cerner toute la complexité du processus de professionnalisation dans lequel s'engagent, au cours des années 1940, ces praticiennes de la santé. Leur quête d'un statut professionnel passe, en effet, par la reconnaissance des compétences qu'elles ont développées et, conséquemment, par une redéfinition de leur rôle et de leur place dans l'univers hospitalier. Cette démarche est cependant porteuse d'enjeux importants puisque reconnaître l'existence de groupes professionnels autonomes oblige non seulement à les rémunérer en conséquence, mais aussi à réaménager les rapports de pouvoir à l'intérieur des institutions hospitalières. Notre étude démontre que ni les administrations hospitalières, ni l'État qui leur succède à titre d'employeur, ni le corps médical ne semblent disposés - pour des motifs différents on l'a vu - à s'engager dans cette voie.

L'intervention de l'État dans le champ de la santé sera déterminante à plusieurs égards. Elle oblige notamment ces trois groupes professionnels à modifier leurs stratégies en ce qui concerne l'amélioration de leurs conditions de travail: malgré certaines réticences, cette main-d'œuvre salariée devra opter pour la voie syndicale, comme l'a fait d'ailleurs la majorité du personnel hospitalier et comme l'ont fait même les médecins en 1963. Cette réorientation permettra de mettre fin en partie à ce «maternage mal salarié» qu'a si bien identifié D. Gaucher en ce qui concerne la main-d'œuvre hospi-

87 CTMQ, Mémoire soumis à la Commission parlementaire des affaires sociales sur le projet de loi 41, septembre 1974. 
talière féminine ${ }^{88}$. C'est, du moins, ce que laissent deviner les premières conventions collectives signées au début des années 1970 par les syndicats de ces trois professions dans le cadre du Cartel des organismes professionnels de la santé (COPS).

Avec l'élaboration d'un nouveau code des professions en 1973, l'État met par ailleurs un terme aux démarches entreprises par ces trois groupes en vue d'obtenir l'exclusivité de leur champ de pratique et d'accroître à la fois leur autonomie professionnelle et leur droit de regard sur la gestion du travail sanitaire. Si cette réorganisation des corps professionnels permet à l'État de mieux gérer la main-d'œuvre hospitalière, elle consolide cependant la structure pyramidale qui caractérise l'univers hospitalier: les catégories d'emploi du domaine paramédical demeurent des professions satellites, œuvrant dans l'orbite du pouvoir médical. Une jonction semble bien s'opérer ici entre les intérêts de l'État devenu employeur et ceux du corps médical. Les physiothérapeutes et les technologistes médicales doivent non seulement composer avec les physiâtres et les médecins de laboratoire, mais aussi avec l'État qui cherchera, surtout pour des raisons de rationalité économique, à limiter leur autonomie professionnelle et leurs pouvoirs dans l'hôpital. Des trois professions étudiées, seule la diététique semble échapper quelque peu à la volonté de contrôle du corps médical. Ceci s'explique peut-être en partie, comme nous l'avons suggéré plus haut, par le fait qu'il n'existe pas encore de spécialisation médicale dans ce domaine. Les diététistes seront néanmoins confrontées au pouvoir de régulation que détient l'État et elles devront lutter pour préserver leur place dans la hiérarchie hospitalière lors de l'étatisation des soins de santé.

En un sens, l'intervention de l'État dans le monde sanitaire constitue un révélateur des rapports de pouvoir qui y prévalent. Devenu partie prenante de ces rapports, l'État constituera désormais un catalyseur de leur transformation en ce sens qu'il confirme, sinon accélère, l'étiolement du modèle libéral comme mode d'organisation des groupes professionnels dans le champ de la santé. Il y a tout lieu de croire qu'à la lumière de cette nouvelle donne, et surtout au lendemain de la réforme du Code des professions, les diététistes, les physiothérapeutes et les technologistes médicales devront, de nouveau, revoir leurs stratégies en vue de l'obtention d'un meilleur statut professionnel. Il s'agit là d'une hypothèse qui mériterait certainement d'être approfondie ${ }^{89}$.

88 D. Gaucher, op. cit.

89 Cette hypothèse alimente d'ailleurs la problématique des recherches que mène présentement notre équipe (Les travailleuses du paramédical au Québec et en Ontario (19201975): le cas des technologistes médicales, des diététistes et des physiothérapeutes). 